\title{
Indicação e uso da ventilação não-invasiva e da cânula nasal de alto fluxo, e orientações sobre manejo da ventilação mecânica invasiva no tratamento da insuficiência respiratória aguda na COVID-19*
}

Indication and use of noninvasive ventilation and high-flow nasal cannula, and guidance on the management of invasive mechanical ventilation for the treatment of acute respiratory failure in COVID-19*

MARTINEZ, Bruno Prata ${ }^{1}$; ANDRADE, Flávio Maciel Dias de ${ }^{2}$; RONCALLI, Ângelo ${ }^{3}$; MARTINS, Jocimar Avelar4; RIBEIRO, Daniel da Cunha ${ }^{5}$; PIANEZZOLA, Ezequiel Mânica ${ }^{6}$; FORGIARINI JÚNIOR, Luiz Alberto; ${ }^{7}$ LIMA, Wildberg Alencar; CAVALHEIRO, Leny Vieira'; MATTE, Darlan Laurício $^{10}$; KARSTEN, Marlus ${ }^{11}$; em nome do Comitê COVID-19 da ASSOBRAFIR.

\section{Resumo}

O presente documento apresenta orientações para o atendimento de Fisioterapia a pacientes com suspeita ou diagnostico de COVID-19, com foco na indicação e uso da ventilação não-invasiva(VNI) e da cânula nasal de alto fluxo (CNAF), bem como sobre manejo da ventilação mecânica invasiva. Algumas intervenções vêm sendo descritas com intuito de melhorar desfechos relacionados à insuficiência respiratória da COVID-19, como a VNI e a CNAF. Com base em estudos científicos atuais, informações advindas da experiência no tratamento da COVID-19, maior risco de disseminação do vírus, aliados à indisponibilidade de alguns recursos na maioria das unidades de terapia intensiva brasileiras, recomenda-se que a VNI e a CNAF não

* Revisado por membros do Comitê COVID-19 da ASSOBRAFIR, nomeado por meio do memorando Nº03/2020. Esta publicação é uma atualização da Comunicação Oficial "Indicação e uso da ventilação não-invasiva e da cânula nasal de alto fluxo, e orientações sobre manejo da ventilação mecânica invasiva no tratamento da insuficiência respiratória aguda na COVID-19", chancelada pelo Comitê COVID-19 da ASSOBRAFIR, originalmente escrita pelos mesmos autores e divulgada em 23/03/2020 no endereço eletrônico https://assobrafir.com.br/covid-19 vni/.

1 Programa de Pós-Graduação em Medicina e Saúde, Universidade Federal da Bahia(UFBA), Salvador, Bahia, Brasil. Colegiado de Fisioterapia, Universidade do Estado da Bahia(UNEB), Salvador, Bahia, Brasil. Email: brunopmartinez@ hotmail.com. BPM - https://orcid.org/ 0000-0002-4673-8698

2 Universidade Católica de Pernambuco, Recife, Pernambuco, Brasil. FMDA - https://orcid.org/0000-0001-9571-6551

${ }_{3}$ Universidade Estadual de Ciências da Saúde de Alagoas, Maceió, Alagoas. AR - https://orcid.org/0000-0002-7944-7168

4 Faculdade Dinâmica do Vale do Piranga, Ponte Nova, Minas Gerais, Brasil. JAM - https://orcid.org/0000-0002-5023-3131

${ }_{6}^{5}$ FISIOUNI - Grupo de Fisioterapia, Brasil. DCR - https://orcid.org/0000-0001-8940-6186

${ }^{6}$ Hospital Niterói D'Or, Niterói, Rio de Janeiro, Brasil. EMP - https://orcid.org/0000-0002-0016-0448

7 Programa de Pós-graduação em Saúde e Desenvolvimento Humano da Universidade La Salle, Canoas, Rio Grande do Sul, Brasil. LAFJ - https://orcid.org/0000-0002-6706-2703

${ }^{8}$ Real Hospital Português de Beneficência em Pernambuco. WAL - https://orcid.org/0000-0002-9584-3961

9 Hospital Israelita Albert Einstein, SP, SP, Brasil. LVC -https://orcid.org/0000-0002-5205-3783

${ }^{10}$ Departamento de Fisioterapia e Programa de Pós-Graduação em Fisioterapia, Universidade do Estado de Santa Catarina (UDESC), Florianópolis, Santa Catarina, Brasil. DLM - https://orcid.org/0000-0003-4650-3714

${ }^{11}$ Departamento de Fisioterapia e Programa de Pós-Graduação em Fisioterapia, Universidade do Estado de Santa Catarina (UDESC), Florianópolis, Santa Catarina, Brasil. Programa de Pós-Graduação em Ciências da Reabilitação, Universidade Federal de Ciências da Saúde de Porto Alegre (UFCSPA), Porto Alegre, Rio Grande do Sul, Brasil. MK - https://orcid.org/0000-0002-2476-7981 
sejam estratégias ventilatórias de primeira linha. Entretanto, em situações específicas, definidas pela equipe multiprofissional, pode ser feito um teste de resposta à VNI ou CNAF, desde que respeitadas condições de biossegurança e resposta do paciente, visando não postergar a intubação orotraqueal.

Palavras-chave: Fisioterapia; Ventilação Não Invasiva(VNI); COVID-19.

\section{Abstract}

This document focus on the use of noninvasive ventilation (NIV) and high-flow nasal cannula (HFNC), as well as on the management of invasive mechanical ventilation in patients with COVID-19. NIV and HFNC have been described to improve outcomes related to the respiratory failure in patients with COVID-19. Based on the available evidence along with a high risk of aerosol dispersion and inadequate environments in most Brazilian intensive care units, NIV and HFNC are not recommended as the first option for ventilatory support in patients with COVID-19. However, a NIV/HFNC trial can be considered once safety measures are guaranteed and according to the patient's response; endotracheal intubation should not be postponed.

Keywords: Physiotherapy; Noninvasive Ventilation; COVID-19.

\section{Objetivo}

O objetivo do presente posicionamento é fornecer orientações para o atendimento de Fisioterapia a pacientes com COVID-19, com foco na indicação e uso da ventilação não-invasiva e da cânula nasal de alto fluxo, bem como sobre manejo da ventilação mecânica invasiva no tratamento da insuficiência respiratória aguda na COVID-19.

\section{Contextualização}

Em janeiro, a ASSOBRAFIR emitiu comunicação sobre o papel do Fisioterapeuta na doença causada por um novo coronavírus (2019-nCoV ou SARS-CoV-2), com informações sobre a nova doença (Coronavirus Disease 2019 ou COVID-19) e orientações gerais sobre a atuação profissional ${ }^{1}$.

Naquela ocasião, as informações disponíveis acerca do tratamento desses pacientes eram bastante escassas e, com base nas orientações disponíveis da Organização Mundial de Saúde (OMS) ${ }^{2}$, a ventilação não invasiva (VNI) foi elencada como possível recurso terapêutico para o tratamento da insuficiência respiratória aguda (IRpA) nos casos da COVID-19.

Entretanto, novas informações foram divulgadas recentemente sobre os desfechos dos pacientes submetidos à VNI e ao uso de cânula nasal de alto fluxo (CNAF), bem como sobre a biossegurança de profissionais de saúde envolvidos na assistência aos pacientes com COVID-19. Assim, entendemos que o aprofundamento daquelas informações era necessário, resultando na atualização apresentada a seguir.

Na comunicação da ASSOBRAFIR, emitida em janeiro/2020르 a indicação da VNI como forma de suporte ventilatório decorreu do fato de a mesma ser amplamente utilizada como recurso para evitar intubação e ventilação mecânica invasiva em pacientes com IRpA hipoxêmica ou hipercápnica, embora os melhores resultados ocorram em pacientes com doença pulmonar obstrutiva crônica e edema pulmonar cardiogênico (EAP) ${ }^{3,4}$. Contudo, a pneumonia viral causada pelo novo coronavírus (SARS-CoV-2), na sua forma grave (Síndrome Respiratória Aguda Severa, SARS), produz hipoxemia grave e refratária à oxigenoterapia, com alterações fisiopatológicas que tem alguma semelhança às encontradas na Síndrome do Desconforto Respiratório Agudo (SDRA). 
Nestes pacientes com SDRA, o subgrupo de pacientes que parece se beneficiar mais da VNI é aquele em que a relação entre pressão parcial de oxigênio no sangue arterial e fração inspirada de oxigênio $\left(\mathrm{PaO}_{2} / \mathrm{FiO}_{2}\right)$ é superior a $200 \mathrm{mmHg}^{5}$. Outros estudos, por sua vez, observaram que o diagnóstico de SDRA foi um dos fatores independentemente associados à falência de $\mathrm{VNI}^{6,7}$. Além disso, em epidemias anteriores, também causadas por pneumonias virais (H1N1 e MERS-COV), assim como na SDRA, os benefícios do emprego da VNI foram bastante limitados ${ }^{8,9}$.

Atualmente, já existe descrição na literatura que o quadro de insuficiência respiratória pela COVID apresenta diferenças em relação à SDRA. Uma das descrições define dois tipos ${ }^{10-11}$ :

- Tipo 1 (Tipo L: low elastance): caracteriza-se por complacência pulmonar relativamente normal; recrutabilidade mínima; hipoxemia devido alteração vascular (perda da vasoconstriç̧ão hipóxica e alteração regulação fluxo de sangue);

- Tipo 2 (Tipo H: high elastance): caracteriza-se por complacência pulmonar diminuída; recrutabilidade maior; hipoxemia devido ao comprometimento de parênquima pulmonar, principalmente devido lesão pulmonar auto-infligida pelo paciente (P-SILI); tomografia com infiltrados difusos bilateral.

Outra definição foi recentemente relatada, na qual existem três tipos, as quais baseiam-se principalmente na análise da tomografia computadorizada de tóra ${ }^{12}$ :

- Tipo 1: caracteriza-se por lesões múltiplas tipo vidro fosco e sinais de hiperperfusão; com complacência normal ou alta;

- Tipo 2: caracteriza-se por atelectasias distribuídas de forma heterogênea, com opacidades peribrônquicas;

- Tipo 3: caracteriza-se pelo padrão clássico da SDRA, com edema alveolar e baixa complacência pulmonar.

No entanto, o conceito de fenótipos proposto nos dois artigos, carece de maior experimentação científica e não deve ser usado de forma isolada como elemento norteador de condutas terapêuticas, já que estes fenótipos descritos podem ser fases da evolução da doença. Essa diferença em relação a SDRA merece atenção, já que isso pode influenciar nos resultados obtidos a depender do tipo ou fase na qual o paciente com COVID-19 se encontra ${ }^{13}$. Recente editorial, descreve que os objetivos antes da intubação orotraqueal devem ser a adequada oxigenação e evitar a P-SILI; já que o esforço respiratório excessivo pode causar estresse pulmonar e vascular, resultando em lesão pulmonar induzida tipo Vortex $^{14}$. Neste material, os autores descrevem que a indicação da VNI ou da CNAF deve ser no período inicial do aumento de trabalho, hipoxemia e alteração radiológica, visando reduzir o esforço muscular e assim impedir o comprometimento importante da complacência pulmonar ao longo do tempo por essa lesão pulmonar ${ }^{10,14}$. No cenário atual de enfrentamento da COVID-19, a VNI variou bastante o seu uso, conforme a localidade ${ }^{15-20}$, com frequências de uso de $11 \%{ }^{15}$ como descrito em recente estudo italiano até $62 \%$ descrito em um estudo chinês ${ }^{20}$. Já em relação a CNAF, a frequência média foi $22,8 \%$ até $63 \% \%^{15-16,18,21-23}$. Neste estudo chinês, onde a CNAF foi utilizada como estratégia de primeira linha em $63 \%$ dos pacientes ${ }^{23}$, seguida de VNI com $33 \%$ e ventilação mecânica invasiva em $4 \%$, o percentual de falha foi $41 \%$ para CNAF, sendo que não houve falha nos pacientes com $\mathrm{PaO}_{2} /$ $\mathrm{FiO}_{2}>200$ e houve $63 \%$ de falha naqueles com $\mathrm{PaO}_{2} / \mathrm{FiO}_{2} \leq 200$. Este último estudo chinês identificou que o uso da CNAF ou VNI como estratégia feita de forma precoce, associada ou não a posição 
prona reduziu necessidade intubação orotraqueal ${ }^{23}$. Em pacientes com insuficiência respiratória hipoxêmica por pneumonias, um recente estudo validou o uso do indíce $\mathrm{ROX}$ (Indíce $\mathrm{ROX}=\left[\mathrm{SpO}_{2} /\right.$ $\left.\mathrm{FiO}_{2}\right] / \mathrm{FR}$ ) como preditor de sucesso da CNAF para não ocorrência de intubação orotraqueal. O valor que demonstrou predição para minimizar risco de intubação orotraqueal foi um indíce de ROX $\geq$ 4,88 nos momentos de 2, 6 e 12 horas. Já os indíces de ROX preditores de falha da CNAF para evitar intubação orotraqueal foram valores $<2,85$ na $2^{\text {a }}$ hora, $<3,47$ na $6^{\text {a }}$ hora e $<3,85$ na $12^{\text {a }}$ hora $^{24}$.

Maiores estudos necessitam avaliar a real eficácia destas intervenções de VNI e CNAF para tratamento da insuficiência respiratória pela COVID-19, já que existe risco de contaminação do ambiente, bem como o esforço muscular pode exacerbar a P-SILI, o que ainda não foi demonstrado na literatura.

O tipo de interface utilizada também é um fator que pode estar associado aos diferentes resultados dos estudos. Patel et al. ${ }^{25}$ compararam a VNI aplicada por meio de capacete (helmet) à utilização da máscara oronasal em 83 pacientes com SDRA, e observaram menor frequência de intubação $(18,2 \%$ vs $61,5 \%$; $\mathrm{p}<0,01)$ e maior taxa de sobrevida em 90 dias $(65,9 \%$ vs $43,6 \%$; $\mathrm{p}=0,02)$ no grupo que utilizou o capacete. Uma metanálise mostrou que a VNI aplicada com o capacete pode reduzir a mortalidade hospitalar, o que não acontece quando são usadas máscaras faciais/nasais 9 . Outra revisão sistemática observou risco três vezes maior de transmissão de infecções respiratórias agudas para profissionais de saúde em procedimentos que geram aerossol, como durante o emprego da $\mathrm{VNI}^{26}$.

De forma complementar, em um estudo de bancada, Hui et al. ${ }^{27}$ demonstraram que o vazamento de ar expirado era insignificante quando a VNI era aplicada por meio de um capacete com circuito de ramos duplos, filtros e boa vedação na interface pescoço-capacete. Por outro lado, o jato de ar exalado pela porta de expiração de uma máscara facial total pode atingir uma distância de aproximadamente $92 \mathrm{~cm}$. Outros estudos também relataram a associação da VNI com a contaminação de trabalhadores da saúde ${ }^{28,29}$.

Vale ressaltar que a aplicação inadequada da VNI pode causar atraso na intubação, o que está diretamente associado ao aumento da mortalidade ${ }^{30,31}$. Em um estudo retrospectivo com 302 pacientes com síndrome respiratória do Oriente Médio (Middle East Respiratory Syndrome, MERS) ${ }^{32}$, observouse que em $35 \%$ deles foi usado incialmente a VNI, sendo que $92 \%$ destes pacientes necessitaram intubação e ventilação mecânica invasiva. Outro estudo com população semelhante mostrou que todos os pacientes submetidos à VNI necessitaram intubação e ventilação por pressão positiva, e que a demora em se ventilar invasivamente pode ter contribuído para o alto índice de óbitos ${ }^{33}$.

No entanto, uma atualização clínica para o tratamento de pacientes com COVID-1934 afirma que em ambientes com acesso limitado à ventilação invasiva ou antes de os pacientes desenvolverem insuficiência respiratória hipoxêmica grave, a VNI ou a CNAF podem ser alternativas úteis. No entanto, como o alto fluxo de gás desses dispositivos é menos contido do que nos circuitos fechados, típicos de ventiladores invasivos, há aumento do risco de dispersão de vírus em aerossol no ambiente assistencial. Assim, a determinação da magnitude desse risco e das estratégias de mitigação dos problemas é um desafio aos profissionais de saúde.

Assim, com base em alguns estudos científicos observacionais atuais ${ }^{15-23}$; nas informações advindas da experiência no tratamento da COVID-1935, que apontam para resultados insatisfatórios e maior risco de disseminação do vírus, aliados à indisponibilidade da interface tipo capacete na 
maioria das unidades de terapia intensiva brasileiras, a ASSOBRAFIR entende que a VNI e a CNAF não devem ser estratégias ventilatórias de primeira linha, destinadas aos pacientes com SARS/ COVID-19.

Entretanto, em situações específicas, definidas pela equipe multiprofissional, pode ser feito um teste de resposta à VNI ou a CNAF, com duração de 30 minutos, nos pacientes com IRpA hipoxêmica. Nessas situações, quando indicada a VNI, devem ser utilizadas máscaras sem válvula de exalação e conectadas a circuitos ventilatórios com ramo duplo. Também deve ser utilizados os filtros trocadores de calor e umidade (FTCU), empregando-se ainda, filtros de barreira nas extremidades distais dos ramos expiratórios dos circuitos ventilatórios, antes das válvulas exalatórias dos ventiladores mecânicos. Quando disponível o aparelho e indicada a CNAF; deve-se existir atenção para o uso dos equipamentos de proteção individual, bem como a necessidade de uma equipe treinada para o uso da intervenção. O paciente deve ser orientado a utilizar mascara cirúrgica para minimizar a dispersão do aerossol. Preferencialmente, esses recursos devem ser aplicados em pacientes alocados em leitos de isolamento respiratório com pressão negativa.

No que concerne a outros pontos relevantes ao manejo ventilatório dos pacientes com as formas severas de pneumonia na COVID-19, submetidos a intubação traqueal, a OMS recomenda algumas medidas visando a redução da incidência de pneumonias bacterianas associadas a ventilação mecânica e redução do risco de disseminação: ${ }^{36}$

- Manutenção do paciente com cabeceira do leito elevada a 30-450: ${ }^{37}$

- Uso de sistema fechado de aspiração em todos os casos;

- Troca dos filtros trocadores de calor e umidade (HME/HMEF) quando observada alteração da sua função, quando sujo, ou a cada intervalo regular de 5 a 7 dias;

- Uso de filtro de barreira (HEPA) na extremidade distal do ramo expiratório do circuito ventilatório, antes da válvula exalatória do ventilador mecânico.

- O uso do filtro HMEF proximal, conectado à máscara, com filtração $>99,9 \%$ substitui o uso dos filtros HME e HEPA.

Após instituição da ventilação mecânica invasiva, deve-se priorizar a adoção da estratégia para minimizar estresse pulmonar e vascular; otimizar oxigenação e impedir a lesão pulmonar tipo vortex ${ }^{14}$. Desta forma, recomenda-se:

- Emprego do modo de ventilação controlada a volume (VCV) ou ventilação controlada a pressão $(\mathrm{PCV}),{ }^{38}$

- Volume corrente alvo: volume corrente ajustado em $6 \mathrm{ml} / \mathrm{Kg}$ ou inferior nos pacientes e pressão de platô inferior a $30 \mathrm{~cm} \mathrm{H20.} \mathrm{No} \mathrm{tipo} \mathrm{1,} \mathrm{é} \mathrm{possível} \mathrm{tolerar} 7$ a $8 \mathrm{ml} / \mathrm{Kg}$, caso haja

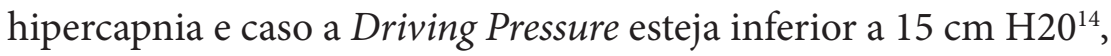

- Manutenção da pressão de distensão alveolar (driving pressure) inferior a $15 \mathrm{cmH} 20^{39}$;

- Os níveis de pressão positiva expiratória final (PEEP) devem ser individualizados, de forma a reduzir os níveis de driving pressure, de forma a reduzir a lesão pulmonar (Níveis abaixo de $15 \mathrm{~cm} \mathrm{H20)}$. Recomenda-se fazer mini-titulação da PEEP a partir de $20 \mathrm{~cm} \mathrm{H20,} \mathrm{com}$ redução de 2 em 2 pontos, para identificação da menor Driving Pressure, sendo mantida a PEEP dois pontos acima da menor Driving Pressure ou no ponto de menor Driving Pressure, 
conforme protocolo da unidade. Nos casos que o aumento do dois pontos da PEEP de menor Driving Pressure e caso a Driving Pressure fique superior a $15 \mathrm{~cm} \mathrm{H} 20$, deve-se usar o PEEP de menor Driving Pressure ou reduzir o volume corrente ${ }^{40-42}$;

- A meta da oxigenação deve ser uma compatível com a vida $\left(\mathrm{PaO}_{2} \geq 60 \mathrm{mmHg}\right.$ e $\mathrm{SpO}_{2}$ $\geq 90 \%)^{43-44}$;

- Tolerância à hipercapnia permissiva; em pacientes com disfunção de ventrículo direito é adequado manter $\mathrm{PaCO}_{2}<50 \mathrm{mmHg}^{45}$.

- A posição prona está indicada para pacientes com relação $\mathrm{PaO}_{2} / \mathrm{FiO}_{2}<150 \mathrm{mmHg}$, devendo ser realizada por equipe multiprofissional devidamente treinada, e mantida por no mínimo 16 horas, caso o paciente apresente resposta satisfatória (aumento de $10 \mathrm{mmHg} \mathrm{na} \mathrm{PaO}_{2}$, ou $20 \mathrm{mmHg}$ na relação $\mathrm{PaO}_{2} / \mathrm{FiO}_{2}$ ). O posicionamento prono deverá ser repetido quando observada uma relação $\mathrm{PaO}_{2} / \mathrm{FiO}_{2}<150 \mathrm{mmHg}$ após 4(quatro) horas em posição supina. Serão considerados como critérios de interrupção do posicionamento prono, reduções de $20 \%$ na relação $\mathrm{PaO} 2 / \mathrm{FiO}_{2}$, após duas tentativas consecutivas de pronação; ${ }^{46}$

- Indicação da manobra de recrutamento alveolar em situações de hipoxemia refratária, não responsiva a outras intervenções, como forma de resgaste;

- Nos casos de hipoxemia severa e refratária, deverá ser discutida com a equipe multiprofissional, a indicação da oxigenação por membrana extracorpórea (ECMO), a qual deve ser, preferencialmente, em centro de referência para a técnica.

No documento publicado pela OMS, também são reforçadas algumas intervenções com foco na redução do tempo de ventilação mecânica, após melhora do quadro agudo ${ }^{36}$ :

- Uso de protocolos de desmame que incluam avaliação diária da capacidade de tolerar a respiração espontânea.

Como orientações relacionadas aos procedimentos de segurança para evitar a contaminação dos profissionais e disseminação do SARS-CoV-2, a OMS recomenda: ${ }^{36}$

- As precauções padrão, que incluem higiene das mãos e uso de equipamentos de proteção individual (EPIs) quando em contato indireto e direto com sangue, fluidos corporais, secreções dos pacientes (incluindo secreções respiratórias) e pele com solução de continuidade (não íntegra). As precauções padrão também incluem a prevenção de ferimentos com agulhas ou objetos cortantes, gerenciamento seguro de resíduos, limpeza e desinfecção de equipamentos, e limpeza do meio ambiente;

- Uso de máscara de proteção N95, ou equivalente, ao entrar em uma sala onde estejam pacientes com suspeita ou confirmação de infecção por SARS-CoV-2, ou em qualquer situação de atendimento prestado a um caso suspeito ou confirmado;

- Uso de máscara de proteção N95, ou equivalente, quando for necessário executar procedimentos que promovam geração de aerossóis.

Além desses pontos apontados pela OMS, sugere-se ainda:

- Idealmente, que os profissionais que atuarem na assistência direta aos casos suspeitos ou confirmados sejam organizados para trabalharem somente na área de isolamento, evitando circulação para outras áreas de assistência. 
- O uso de óculos de proteção ou protetores faciais (que cubram a frente e os lados do rosto); aventais impermeáveis de mangas longas, punho de malha ou elástico e abertura posterior; e luvas, que deverão ser utilizados durante a assistência destinada a qualquer paciente com suspeita, ou confirmação de infecção pelo SARS-CoV-2. Estes devem ser de uso exclusivo de cada profissional responsável pela assistência, sendo necessária a higiene correta, ou descarte após o uso.

\section{Considerações Finais}

Considerando a necessidade de evitar uma maior disseminação para o meio durante as intervenções, bem como a indicação de intervenções que gerem melhora dos resultados e desfechos clínicos, a indicação da VNI e da CNAF deverá ser feita com base em minuciosa e específica avaliação pelo fisioterapeuta e demais membros da equipe multiprofissional, bem como da disponibilidade de equipamentos e ambiente necessário.

Com base em alguns estudos científicos observacionais atuais; nas informações advindas da experiência no tratamento da COVID-19, que apontam para resultados insatisfatórios, alta taxa de falha e maior risco de disseminação do vírus, aliados à indisponibilidade da interface tipo capacete na maioria das unidades de terapia intensiva brasileiras, não recomendamos a VNI e a CNAF como estratégias ventilatórias de primeira linha, destinadas aos pacientes com SARS/COVID-19.

Poucas evidências estão disponíveis até o momento sobre a segurança, momento ideal e real efetividade da VNI e da CNAF em pacientes com COVID-19; logo, as recomendações aqui apresentadas se baseiam principalmente na opinião de especialistas e nos direcionamentos fornecidos por entidades nacionais e internacionais.

\section{Referências}

1. Matte DL, Andrade FMD, Martins JA, Martinez BP, Karsten M. O fisioterapeuta e sua relação com o novo betacoronavirus 2019 (2019-nCoV): comunicação oficial da ASSOBRAFIR [Internet]. São Paulo: ASSOBRAFIR; 2020. Available from: https://assobrafir.com.br/assobrafir betacoronavirus2019/2020.

2. WHO. Infection prevention and control during health care when novel coronavirus (nCoV) infection is suspected. Interim guidance 25 January 2020 [Internet]. Geneva: World Health Organization; 2020. Available from: https://apps.who.int/iris/handle/10665/330674.

3. Weng C-L, Zhao Y-T, Liu Q-H, Fu C-J, Sun F, Ma Y-L, et al. Meta-analysis: Noninvasive Ventilation in Acute Cardiogenic Pulmonary Edema. Ann Intern Med. 2010 May 4;152(9):590-600. doi: 10.7326/0003-4819-152-9-201005040-00009.

4. Osadnik CR, Tee VS, Carson-Chahhoud KV, Picot J, Wedzicha JA, Smith BJ. Non-invasive ventilation for the management of acute hypercapnic respiratory failure due to exacerbation of chronic obstructive pulmonary disease. Cochrane Database Syst Rev. 2017 Jul 13;7(7):CD004104. doi: 10.1002/14651858.CD004104.pub4.

5. Zhan Q, Sun B, Liang L, Yan X, Zhang L, Yang J, et al. Early use of noninvasive positive pressure ventilation for acute lung injury: A multicenter randomized controlled trial. Crit Care Med. 2012 Feb;40(2):455-60. doi: 10.1097/CCM.0b013e318232d75e. 
6. Antonelli M, Conti G, Moro M, Esquinas A, Gonzalez-Diaz G, Confalonieri M, et al. Predictors of failure of noninvasive positive pressure ventilation in patients with acute hypoxemic respiratory failure: a multi-center study. Intensive Care Med. 2001 Nov;27(11):1718-28. doi: 10.1007/s00134001-1114-4. Epub 2001 Oct 16.

7. Adda M, Coquet I, Darmon M, Thiery G, Schlemmer B, Azoulay É. Predictors of noninvasive ventilation failure in patients with hematologic malignancy and acute respiratory failure. Crit Care Med. 2008 Oct;36(10):2766-72. doi: 10.1097/CCM.0b013e31818699f6.

8. Agarwal R, Aggarwal AN, Gupta D. Role of Noninvasive Ventilation in Acute Lung Injury/Acute Respiratory Distress Syndrome: A Proportion Meta-analysis. Respir Care. 2010 Dec;55(12):1653-60.

9. Xu X-P, Zhang X-C, Hu S-L, Xu J-Y, Xie J-F, Liu S-Q, et al. Noninvasive Ventilation in Acute Hypoxemic Nonhypercapnic Respiratory Failure: A Systematic Review and Meta-Analysis. Crit Care Med. 2017 Jul;45(7):e727-e733. doi: 10.1097/CCM.0000000000002361.

10. Gattinoni L, Chiumello D, Caironi P, Busana M, Romitti F, Brazzi L, et al. COVID-19 pneumonia: different respiratory treatments for different phenotypes?. Intensive Care Med. 2020 Jun;46(6):10991102. doi: 10.1007/s00134-020-06033-2. Epub 2020 Apr 14.

11. Gattinoni L, Chiumello D, Rossi S. COVID-19 pneumonia: ARDS or not? Crit Care. 2020 Apr 16;24(1):154. doi: 10.1186/s13054-020-02880-z.

12. Robba C, Battaglini D, Ball L, Patroniti N', Loconte M, Brunetti I, et al. Distinct phenotypes require distinct respiratory management strategies in severe COVID-19. Respir Physiol Neurobiol. 2020 Aug;279:103455. doi: 10.1016/j.resp.2020.103455. Epub 2020 May 11.

13. Bos LDJ, Sinha P, Dickson RP. The perils of premature phenotyping in COVID: a call for caution. Eur Respir J. 2020 Jul 23;56(1):2001768. doi: 10.1183/13993003.01768-2020.

14. Marini JJ, Gattinoni L. Managemtnet of COVID-19 Respiratory Distress. JAMA. 2020 Apr 24. doi: 10.1001/jama.2020.6825. Online ahead of print.

15. Grasselli G, Zangrillo A, Zanella A, Antonelli M; Cabrini L; Castelli A, et al. Baseline Characteristics and Outcomes of 1591 Patients Infected With SARS-CoV2 Admitted to ICU sof the Lombardy Region, Italy. JAMA. 2020 Apr 6;323(16):1574-1581.doi: 10.1001/jama.2020.5394. Online ahead of print.

16. Arentz M, Yim E, Klaff L, Lokhandwala S, Riedo FX, Chong M, et al. Characteristics and outcomes of 21 critically ill patients with COVID-19 in Washington State. JAMA. 2020 Mar 19;323(16):16121614. doi: 10.1001/jama.2020.4326. Online ahead of print.

17. Wang D, Hu B, Hu C, Zhu F, Liu X, Zhang J, et al. Clinical characteristics of 138 hospitalized patients with 2019 novel coronavirus-infected pneumonia in Wuhan, China. JAMA. 2020 Feb 7;323(11):1061-1069. doi: 10.1001/jama.2020.1585. Online ahead of print.

18. Yang X, Yu Y, Xu J, Shu H, Xia J, Liu H, et al. Clinical course and outcomes of critically ill patients with SARS-CoV-2 pneumonia in Wuhan, China: a single-centered, retrospective, observational study. Lancet Respir Med. 2020 May;8(5):475-481. doi: 10.1016/S2213-2600(20)30079-5. Epub 2020 Feb 24.

19. Zhou F, Yu T, Du R, Fan G, Liu Y, Liu Z, et al. Clinical course and risk factors for mortality of adult in patients with COVID-19 in Wuhan, China: a retrospective cohort study. Lancet. 2020 Mar 28;395(10229):1054-1062. doi: 10.1016/S0140-6736(20)30566-3. Epub 2020 Mar 11.

20. Huang C, Wang Y, Li X, Ren L, Zhao J, Hu Y, et al. Clinical features of patients infected with 2019 novel coronavirus in Wuhan, China. Lancet. 2020 Feb 15;395(10223):497-506. doi: 10.1016/S01406736(20)30183-5. Epub 2020 Jan 24. 
21. Chen T, Wu D, Chen H, Yan W, Yang D, Chen G, et al. Clinical characteristics of 113 deceased patients with coronavirus disease 2019: retrospective study. BMJ. 2020 Mar 26;368:m1091. doi: 10.1136/bmj.m1091.

22. Bhatraju PK, Ghassemieh BJ, Nichols M, Kim R, Jerome KR, Nalla AK, et al. Covid-19 in critically ill patients in the Seattle region - Case Series. N Engl J Med. 2020 May 21;382(21):2012-2022. doi: 10.1056/NEJMoa2004500. Epub 2020 Mar 30.

23. Wang K, Zhao W, Li J, Shu W, Duan J. The experience of high-flow nasal cannula in hospitalized patients with 2019 novel coronavirus-infected pneumonia in two hospitals of Chongqing, China. Ann Intensive Care. 2020 Mar 30;10(1):37. doi: 10.1186/s13613-020-00653-z.

24. Roca O, Caral B, Messika J, Samper M, Sztrymf B, Hernandez G, et al. An Index Combining Respiratory Rate and Oxygenation to Predict Outcome of Nasal High-Flow Therapy. m J Respir Crit Care Med. 2019 Jun 1;199(11):1368-1376. doi: 10.1164/rccm.201803-0589OC.

25. Patel BK, Wolfe KS, Pohlman AS, Hall JB, Kress JP. Effect of Noninvasive Ventilation Delivered by Helmet vs Face Mask on the Rate of Endotracheal Intubation in Patients With Acute Respiratory Distress Syndrome: A Randomized Clinical Trial. JAMA. 2016 Jun 14;315(22):2435-41. doi: 10.1001/ jama.2016.6338.

26. Tran K, Cimon K, Severn M, Pessoa-Silva CL, Conly J. Aerosol generating procedures and risk of transmission of acute respiratory infections to healthcare workers: a systematic review. PLoS One. 2012;7(4):e35797. doi: 10.1371/journal.pone.0035797. Epub 2012 Apr 26.

27. Hui DS, Chow BK, Lo T, Ng SS, Ko FW, Gin T, et al. Exhaled Air Dispersion During Noninvasive Ventilation via Helmets and a Total Facemask. Chest. 2015 May;147(5):1336-1343. doi: 10.1378/ chest.14-1934.

28. Fowler RA, Guest CB, Lapinsky SE, Sibbald WJ, Louie M, Tang P, et al. Transmission of Severe Acute Respiratory Syndrome during Intubation and Mechanical Ventilation. Am J Respir Crit Care Med. 2004 Jun 1;169(11):1198-202. doi: 10.1164/rccm.200305-715OC. Epub 2004 Feb 27.

29. Xiao Z, Li Y, Chen R, Li S, Zhong S, Zhong N. A retrospective study of 78 patients with severe acute respiratory syndrome. Chin Med J (Engl). 2003 Jun;116(6):805-10.

30. Nava S, Schreiber A, Domenighetti G. Noninvasive Ventilation for Patients With Acute Lung Injury or Acute Respiratory Distress Syndrome. Respir Care. 2011 Oct;56(10):1583-8. doi: 10.4187/ respcare.01209.

31. Hraiech S, Alingrin J, Dizier S, Brunet J, Forel J-M, La Scola B, et al. Time to intubation is associated with outcome in patients with community-acquired pneumonia. PLoS One. 2013 Sep 19;8(9):e74937. doi: 10.1371/journal.pone.0074937. eCollection 2013.

32. Alraddadi BM, Qushmaq I, Al-Hameed FM, Mandourah Y, Almekhlafi GA, Jose J, et al. Noninvasive ventilation in critically ill patients with the Middle East respiratory syndrome. Influenza Other Respir Viruses. 2019 Jul;13(4):382-390. doi: 10.1111/irv.12635. Epub 2019 Mar 18.

33. Arabi YM, Arifi AA, Balkhy HH, Najm H, Aldawood AS, Ghabashi A, et al. Clinical Course and Outcomes of Critically Ill Patients With Middle East Respiratory Syndrome Coronavirus Infection. Ann Intern Med. 2014 Mar 18;160(6):389-97. doi: 10.7326/M13-2486.

34. Murthy S, Gomersall CD, Fowler RA. Care for Critically Ill Patients With COVID-19. JAMA. 2020 Mar 11. doi: 10.1001/jama.2020.3633. Online ahead of print.

35. Cheung JC, Ho LT, Cheng JV, Cham EYK, Lam KN. Staff safety during emergency airway 
management for COVID-19 in Hong Kong. Lancet Respir Med. 2020 Apr;8(4):e19. doi: 10.1016/ S2213-2600(20)30084-9. Epub 2020 Feb 24.

36. World Health Organization. Clinical management of severe acute respiratory infection (SARI) when COVID-19 disease is suspected: interim guidance, 13 March 2020 [Internet]. Geneva: World Health Organization; 2020. Available from: https://apps.who.int/iris/handle/10665/331446.

37. Wang L, Li X, Yang Z, Tang X, Yuan Q, Deng L, Sun X. Semi-recumbent position versus supine position for the prevention of ventilator-associated pneumonia in adults requiring mechanical ventilation. Cochrane Database Syst Rev. 2016 Jan 8;2016(1):CD009946. doi: 10.1002/14651858. CD009946.pub2.

38. Chacko B, Peter JV, Tharyan P, John G, Jeyaseelan L. Pressure-controlled versus volume-controlled ventilation for acute respiratory failure due to acute lung injury (ALI) or acute respiratory distress syndrome (ARDS). Cochrane Database Syst Rev. 2015 Jan 14;1:CD008807. doi: 10.1002/14651858. CD008807.pub2.

39. Amato MB, Meade MO, Slutsky AS, Brochard L, Costa EL, Schoenfeld DA, et al. Driving pressure and survival in the acute respiratory distress syndrome. N Engl J Med. 2015 Feb 19;372(8):747-55. doi: 10.1056/NEJMsa1410639.

40. AMIB. Recomendações da Associação de Medicina Intensiva Brasileira para a abordagem do COVID-19 em medicina intensiva. 2020

41. Kacmarek RM, Villar J, Sulemanji D, Montiel R, Ferrando C, Blanco J, et al. Open Lung Approach for the Acute Respiratory Distress Syndrome: A Pilot, Randomized Controlled Trial. Crit Care Med. 2016 Jan;44(1):32-42. doi: 10.1097/CCM.0000000000001383.

42. Sahetya SK, Hager DN, Stephens RS, Needham DM, Brower RG. PEEP Titration to Minimize Driving Pressure in Subjects With ARDS: A Prospective Physiological Study. Respir Care. 2020 May;65(5):583-589. doi: 10.4187/respcare.07102. Epub 2019 Nov 26.

43. ICU-ROX Investigators and the Australian and New Zealand Intensive Care Society Clinical Trials Group, Mackle D, Bellomo R, Bailey M, Beasley R, Deane A, et al. Conservative oxygen therapy during mechanical ventilation in the ICU. N Engl J Med. 2020 Mar 12;382(11):989-998. doi: 10.1056/ NEJMoa1903297. Epub 2019 Oct 14.

44. Barrot L, Asfar P, Mauny F, Winiszewski H, Montini F, Badie J, et al. Liberal or Conservative Oxygen Therapy for Acute Respiratory Distress Syndrome. N Engl J Med. 2020 Mar 12;382(11):9991008. doi: 10.1056/NEJMoa1916431.

45. Repessé X, Vieillard-Baron A. Right heart function during acute respiratory distress syndrome. Ann Transl Med. 2017 Jul;5(14):295. doi: 10.21037/atm.2017.06.66.

46. Guérin C, Reignier J, Richard J, Beuret P, Gacouin A, Boulain T, et al. Prone Positioning in Severe Acute Respiratory Distress Syndrom. N Engl J Med. 2013 Jun 6;368(23):2159-68. doi: 10.1056/ NEJMoa1214103. Epub 2013 May 20.

Submissão em: 22/06/2020

Aceite em: 15/07/2020 